\title{
Progress and prospects toward our understanding of the evolution of dosage compensation
}

\author{
Beatriz Vicoso • Doris Bachtrog
}

Published online: 23 July 2009

(C) The Author(s) 2009. This article is published with open access at Springerlink.com

Keywords Dosage compensation · Sex chromosomes $\cdot$ Evolution $\cdot$ Chromatin $\cdot$ Epigenetics
Abbreviations
ChIP-seq
C. elegans
D. busckii
D. melanogaster
D. miranda
DCC
DNA
dox
DPY
LINE
$\operatorname{Lnx}$
LTR
MIX
mle
MOF
mRNA
MSL
POF
PRC
rex
RNA
roX
SDC
Chromatin ImmunoPrecipitation and Sequencing
Caenorhabditis elegans
Drosophila buskii
Drosophila melanogaster
Drosophila miranda
Dosage Compensation Complex
DeoxyRibonucleic Acid
Dependent On X
DumPY
Long Interspersed Elements
Ligand of Numb-protein X
Long Terminal Repeat
MItosis and $\mathrm{X}$ associated
Maleless
Males absent On the First
Messenger DeoxyRibonucleic
Acid
Male-Specific Lethal
Painting Of Fourth
Polycomb Repressive Complex
Recruitment Elements on X
RiboNucleic Acid
RNA on the $\mathrm{X}$
Sex determination and Dosage
Compensation defect 


$\begin{array}{ll}\text { SMC } & \text { Structural Maintenance of } \\ & \text { Chromosomes } \\ \text { Sxl } & \text { Sex Lethal } \\ \text { TE } & \text { Transposable Element } \\ \text { Tsix } & \text { Xist antisense RNA (Xist spelt } \\ & \text { backwards) } \\ \text { XIC } & \text { X Inactivation Center } \\ \text { Xist } & \text { X inactive specific transcript }\end{array}$

\section{The evolution of heteromorphic sex chromosomes and the pressure to increase $\mathrm{X}$-linked expression in males}

Species with separate sexes are widespread among animals and plants. Sex determination can vary widely within clades, with mechanisms as different as environmental and genetic determination occurring in related species, which indicates frequent de novo evolution of sex-determining mechanisms (Bull 1983). Heteromorphic sex chromosomes - such as the XY pair of mammals - have arisen independently from ancestral pairs of autosomes several times and display very similar characteristics, suggesting that their evolution followed similar steps, outlined below.

Y-chromosomes can arise when a male-determining gene appears on an autosome (in the case of male heterogamety, such as the mammalian system; the same principles apply in the case of female heterogamety; Charlesworth 1996). From that moment on, this newly formed sex chromosome (the proto-Y chromosome) is always transmitted to males; mutations that favour males are therefore predicted to accumulate there, even if they have deleterious effects in females. When a recombination event occurs between the proto- $\mathrm{Y}$ and the proto-X chromosomes, however, some of these male-beneficial mutations are relocated to the $\mathrm{X}$, where they may be selected against in females. Selection therefore favours reduced recombination on the proto-Y chromosome to protect linkage between the maledetermining gene and male-beneficial genes.

This reduced or absent recombination on the $\mathrm{Y}$ incurs a long-term cost for males: by reshuffling loci during meiosis, recombination creates a wide range of allele combinations for natural selection to act upon, thereby increasing the efficacy of natural selection (Hill and Robertson 1966). In the absence of recombination, all the sites on the chromosome are completely linked, forcing selection to act on a limited range of allele combinations. This has two main consequences: first, whenever a beneficial mutation is fixed in the population, all deleterious mutations located on the $\mathrm{Y}$ chromosome that carries the advantageous allele will become fixed simultaneously (Rice 1987). Second, purifying selection against mildly or moderately deleterious mutations will become less efficient on a non-recombining chromosome, resulting in their gradual accumulation on the Y chromosome (Charlesworth 1996). These evolutionary theories predict that after recombination is repressed, deleterious mutations in protein-coding genes and regulatory regions, and repetitive DNAsuch as transposable elements - will quickly accumulate on the proto-Y. In species with relatively ancient sex chromosomes, like mammals or D. melanogaster, the $\mathrm{Y}$ chromosome has lost most of its original genes and consists to a large extent of repetitive junk DNA (Lahn et al. 2001). Genomic data from species that have only recently acquired their sex chromosomes have shown that the $\mathrm{Y}$ chromosome shows higher rates of amino-acid substitution (probably reflecting an accumulation of deleterious mutations) and frame-shift mutations, an accumulation of transposable elements and disrupted patterns of gene expression (Guttman and Charlesworth 1998; Charlesworth 2004; Liu et al. 2004; Bachtrog 2006; Bachtrog et al. 2008).

\section{Different strategies of solving the dosage problem: up- and down-regulation of $\mathrm{X}$ chromosomes in mammals, worms and flies}

As genes on the $\mathrm{Y}$ chromosome degenerate, males are left with only one functional copy of X-Y genes, leading to imbalances of $\mathrm{X}$-linked versus autosomal gene expression. This favours the evolution of mechanisms that increase the expression of genes on the single male $\mathrm{X}$ chromosome, i.e. dosage compensation (Charlesworth 1978; Engelstädter 2008). Dosage compensation was first discovered in $D$. melanogaster by $\mathrm{H}$. Muller and has been extensively studied in Drosophila, Caenorhabditis, and mammals (reviewed in Straub and Becker 2007; see Fig. 1). In Drosophila, dosage compensation is achieved by doubling the expression of $\mathrm{X}$-linked genes in males, using a male-specific ribonucleoprotein complex. 

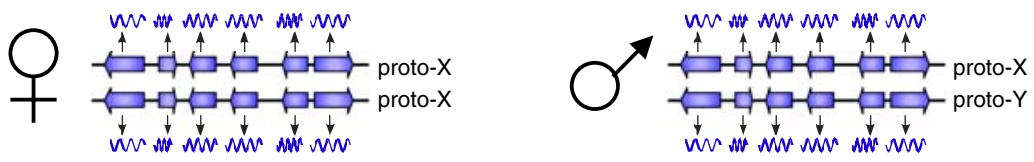

Y degeneration
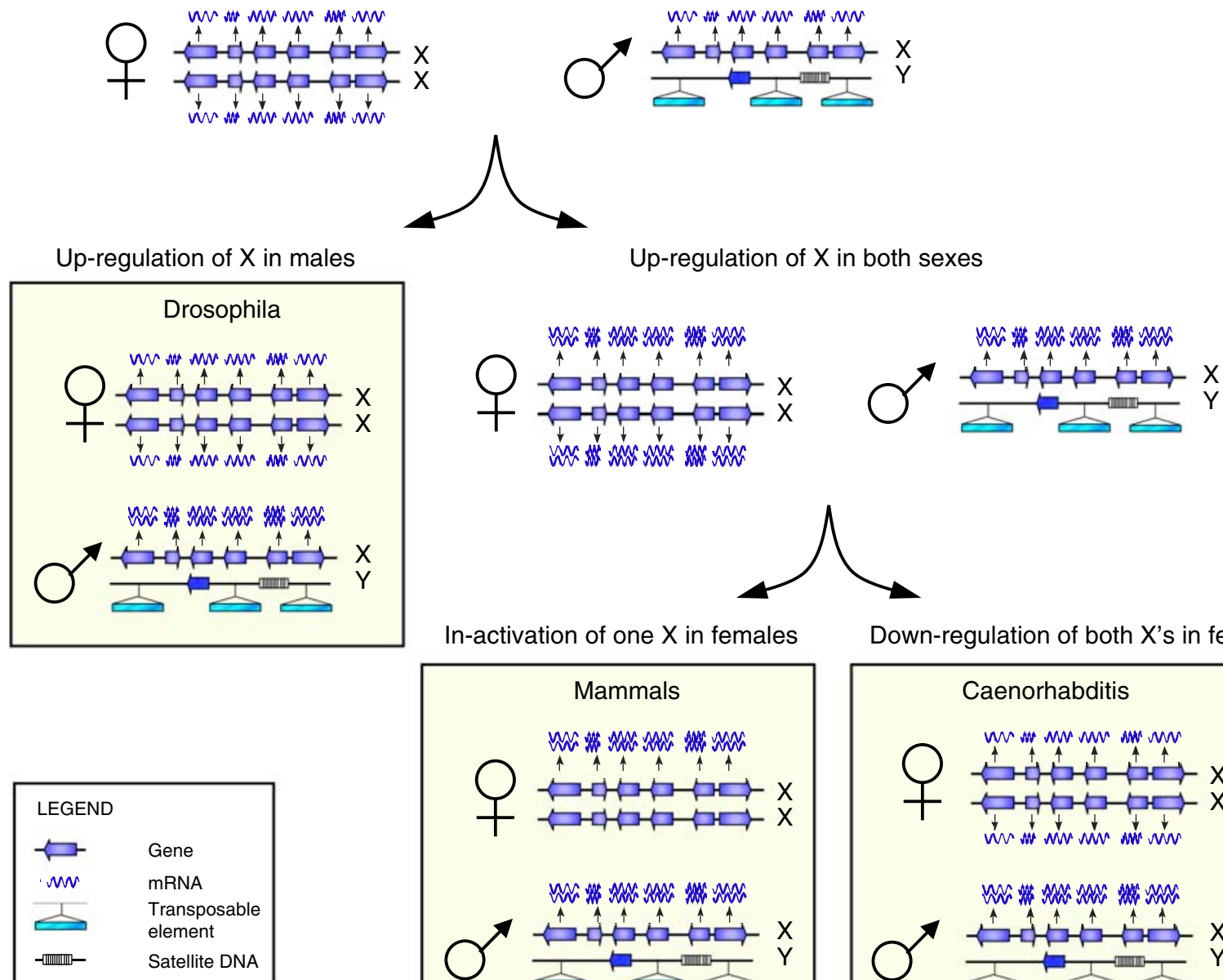

In-activation of one $X$ in females

Down-regulation of both X's in females
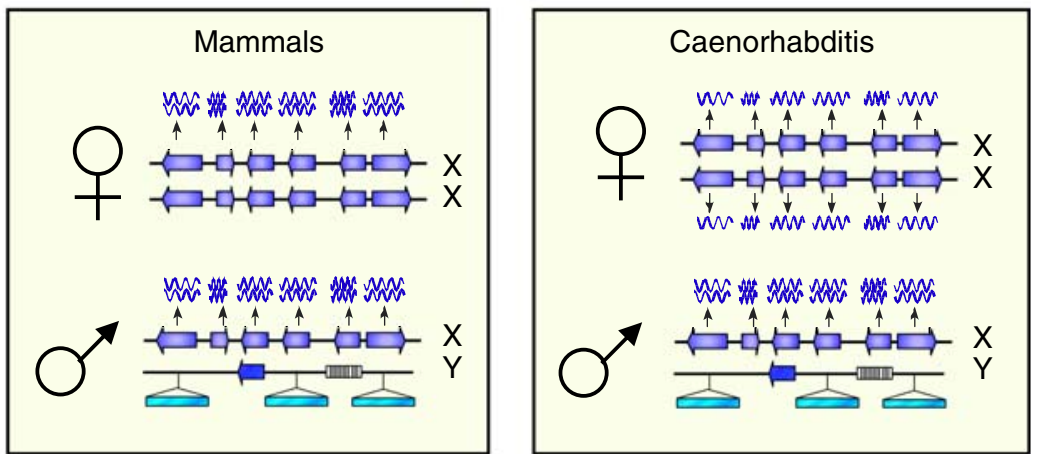

Fig. 1 The evolution of dosage compensation in flies, mammals and worms. Sex chromosomes originated independently many times from ordinary autosomes, and Y degeneration is a general facet of sex chromosome evolution (i.e. mutational melt-down of genes and accumulation of transposable elements and satellite DNA). Selective pressure to restore expression levels of X-linked genes relative to autosomes in males accompanies Y-chromosome degeneration, thus driving the evolution of dosage compensation. In Drosophila, dosage compensation is achieved by up-regulation of X-linked genes in males only, and no further modification of Xlinked expression is necessary. In mammals and C. elegans, the $\mathrm{X}$ has become up-regulated in both sexes. While this ensures proper expression balance between $\mathrm{X}$-linked and autosomal genes in males, the $\mathrm{X}$ will be over-transcribed in females, and secondary mechanisms have evolved to restore proper gene dose in females. In mammals, one $\mathrm{X}$-chromosome has become completely inactivated, while Caenorhabditis halves expression from both of its $\mathrm{X}$ chromosomes in hermaphrodites 
Under this form of dosage compensation, no secondary adjustments of expression levels of X-linked genes in females are necessary (Fig. 1).

The classical view of dosage compensation in mammals and $C$. elegans is that females halve the expression of $\mathrm{X}$-linked genes to equalize gene expression between the sexes. Evolutionary biologists, however, noted early on that selection acts on individuals, so that a pressure to equalize expression differences between the sexes cannot account for the evolution of these compensation mechanisms. Furthermore, simple down-regulation of the $\mathrm{X}$ in females would only exacerbate the $\mathrm{X} /$ autosomal expression dosage difference, since dosage imbalances would then affect females as well as males (Gupta et al. 2006). Thus, it was suggested that there is in fact up-regulation of expression of $\mathrm{X}$-linked genes, but that unlike in Drosophila, this up-regulation of X-linked genes was not male-specific but occurred in both sexes. In order to restore correct gene dose between the $\mathrm{X}$ and autosomes in females, repression of X-linked expression in females would have to evolve secondarily (Fig. 1). Charlesworth (1978; 1996) presented a verbal sketch of this model, and it was recently modelled theoretically. Assuming that males with a single active allele have suboptimal levels of X-linked expression and females with two active copies have the optimal level of gene expression, Engelstädter and Haig (2008) explored under which conditions increased X-linked gene expression in both sexes followed by $\mathrm{X}$ inactivation in females can evolve. They showed that up-regulation of X-linked genes in both sexes can evolve, as long as the fitness reduction in females is small relative to the fitness increase in males. Experiments in Drosophila support the notion that the deleterious effects of gene dosage reductions are generally more pronounced than those of similarly sized gene dosage increases (Lindsley et al. 1972). The theoretical prediction that inactivation or downregulation of the $\mathrm{X}$ in females has evolved in response to up-regulation of the $\mathrm{X}$ in both sexes has recently been confirmed through exhaustive comparisons of $\mathrm{X}$-linked and autosomal expression levels in mouse, humans and C. elegans. In particular, expression from $\mathrm{X}$-linked genes in all sampled tissues is, on average, approximately equal to that from autosomal genes both in females and in males (Gupta et al. 2006; Nguyen and Disteche 2006; Lin et al. 2007). The actual mechanism behind this up-regulation of $\mathrm{X}$ - linked genes in both sexes is unknown, and molecular studies so far have focused on understanding the pathways controlling female repression of X-linked over-expression. It is possible that X-linked genes are selected individually for promoters with increased activity; however, examination of expression profiles in mouse germ cells and early zygotes suggests that up-regulation is absent from haploid germ cells but is rapidly established upon fertilization, suggesting a more elaborate, chromosome-wide regulatory mechanism (Nguyen and Disteche 2006).

\section{Molecular mechanisms of dosage compensation: the importance of chromatin modifying complexes}

While the mechanisms of dosage compensation differ markedly between taxa, each well-studied case involves the recruitment of a chromatin regulatory complex to modulate the expression on the entire $\mathrm{X}$ chromosome (Straub and Becker 2007). In D. melanogaster, a male-specific RNA-protein complex-the dosage compensation complex (DCC) - binds to the male $\mathrm{X}$ chromosome and doubles its expression, thereby readjusting the $\mathrm{X}$ to autosomal expression ratio. The DCC requires at least five "male-specific lethal" (MSL) proteins ( $m s l-1, m s l-2, m s l-3, m l e$, and $m o f$ ), and two (functionally redundant) non-coding RNAs, roX1 and roX2 (Park and Kuroda 2001). The DCC (or MSL-complex) binds almost exclusively to specific sites along the $\mathrm{X}$ chromosome, where it interferes with chromatin folding by acetylating lysine 16 of $\mathrm{H} 4$, a histone required for the folding of nucleosomal arrays into $30 \mathrm{~nm}$ fibres, possibly making the genetic material more readily accessible to the transcription machinery (Park and Kuroda 2001).

As noted above, the mechanisms resulting in nonsex-specific up-regulation of X-linked genes in mammals and worms are unknown, and molecular studies have instead concentrated on understanding the inactivation or down-regulation of X-linked transcription in females (or hermaphrodites). Mammals achieve down-regulation of X-linked genes in females by abolishing transcription of one copy of the X chromosome in each cell (Lyon 1961), through a process known as $\mathrm{X}$-inactivation (see Payer and Lee 2008 for a detailed review of the molecular mechanisms involved). X-inactivation is controlled by one $\mathrm{X}$-linked locus only, the X-inactivation center (XIC), 
which contains the two non-coding RNAs directly involved in mediating $\mathrm{X}$ chromosome inactivation, Xist and Tsix. Xist and Tsix are antisense RNAs (they originate from the same locus, but are transcribed in opposite directions) and function as mutual repressors of transcription. The Tsix copy located on the inactive $\mathrm{X}$ chromosome is repressed, so that Xist is highly transcribed and coats the $\mathrm{X}$ chromosome, recruiting the proteins that cause its heterochromatic state; on the active X, Tsix is expressed, and Xist expression is repressed. Two Polycomb repressive complexes, PRC1 and PRC2, are recruited to the X chromosome that is coated with Xist and modify histones, leading to a stably inactivated X (Straub and Becker 2007).

C. elegans females (or more accurately, XX hermaphrodites) have adopted a similar strategy to mammals, but halve the transcription rate of each $\mathrm{X}$-chromosome instead of completely inactivating one copy (Meyer and Casson 1986). This requires the action of a dosage compensation complex formed by at least nine proteins, several of which are also involved in sex-determination (the sex determination and dosage compensation genes, or SDC genes). All the genes that form the $C$. elegans DCC are supplied maternally to both $\mathrm{XX}$ and $\mathrm{XO}$ embryos, with one exception: SDC-2 is expressed exclusively in $\mathrm{XX}$ embryos, where it mediates both the development of a hermaphroditic phenotype and the recruitment of the DCC proteins to the DCC (Dawes et al. 1999). A subset of these proteins then form a condensin-like subdomain, which is thought to be responsible for the chromatin modifications that cause the decreased expression levels on the $\mathrm{X}$ (Hagstrom and Meyer 2003).

\section{Recognition of the $\mathrm{X}$ chromosome: cis-elements and spreading}

One aspect of the process of dosage compensation that remained elusive until recently is the specific targeting of the X chromosome by the DCC. Recent studies point to mechanisms of dosage compensation that combine recognition and targeting of the $\mathrm{X}$ chromosome by the DCC which involves degenerate DNA sequence motifs together with spreading of modifications in cis from recognition sites, resulting in complete coating of the X chromosome by the DCC. However, the relative importance of targeting versus spreading is surprisingly different between flies, worms and mammals (Fig. 2). The simplest targeting mechanism exists in humans, where X-inactivation is controlled by a single X-linked locus only-the X-inactivation center (XIC) - which directs silencing to flanking chromatin (Fig. 2). Interestingly, translocations of the XIC to an autosome lead to at least partial inactivation of the XIC-carrying autosome, suggesting that no further sequence signalling along the chromosome is essential. However, while silencing of autosomal chromatin will occur, silencing does not spread as far or repress as stably as when it occurs on the X chromosome (White et al. 1998). Thus, X-linked sequence elements that promote the spread and maintenance of silencing have been proposed, and a candidate sequence that is enriched on the $\mathrm{X}$ and facilitates spread of silencing in X:A translocations is a class of LINE elements. It has been suggested that in humans as many as $15 \%$ of $\mathrm{X}$-linked genes consistently escape X-inactivation (Carrel and Willard 2005); thus, regions of the $\mathrm{X}$ chromosome that escape X-inactivation would need to have acquired additional signals that repress the spreading of the inactivation machinery. However, escape from $\mathrm{X}$ inactivation in humans can, to a large extent, be explained by the evolutionary history of the sex chromosomes. Several "evolutionary strata" have been identified on the mammalian sex chromosomes, which correspond to different time points of recombination suppression between the proto-sex chromosomes. Almost all of the human genes in the oldest stratum are inactivated, and most of the genes that escape $\mathrm{X}$ inactivation are located in the most recent stratum. This supports the idea that secondary Xist regulating sites are located along the $\mathrm{X}$ chromosome to modulate and stabilize Xist-binding.

Recognition of the $\mathrm{X}$ in Drosophila also follows a two-step model (Kelley et al. 1999); however, unlike in mammals, many more chromosome entry sites that recruit the DCC to the X chromosome in a sequencedependent manner have been identified, and spreading occurs over much smaller distances (Fig. 2). Initial suggestions that the $\mathrm{X}$ chromosome of Drosophila possesses only 35-40 high-affinity "entry sites" that the DCC recognizes and binds to, followed by spreading in cis along the entire chromosome, were experimentally supported by translocations of identified $\mathrm{X}$ chromosome entry points onto autosomes. This led to DCC binding not only to the 


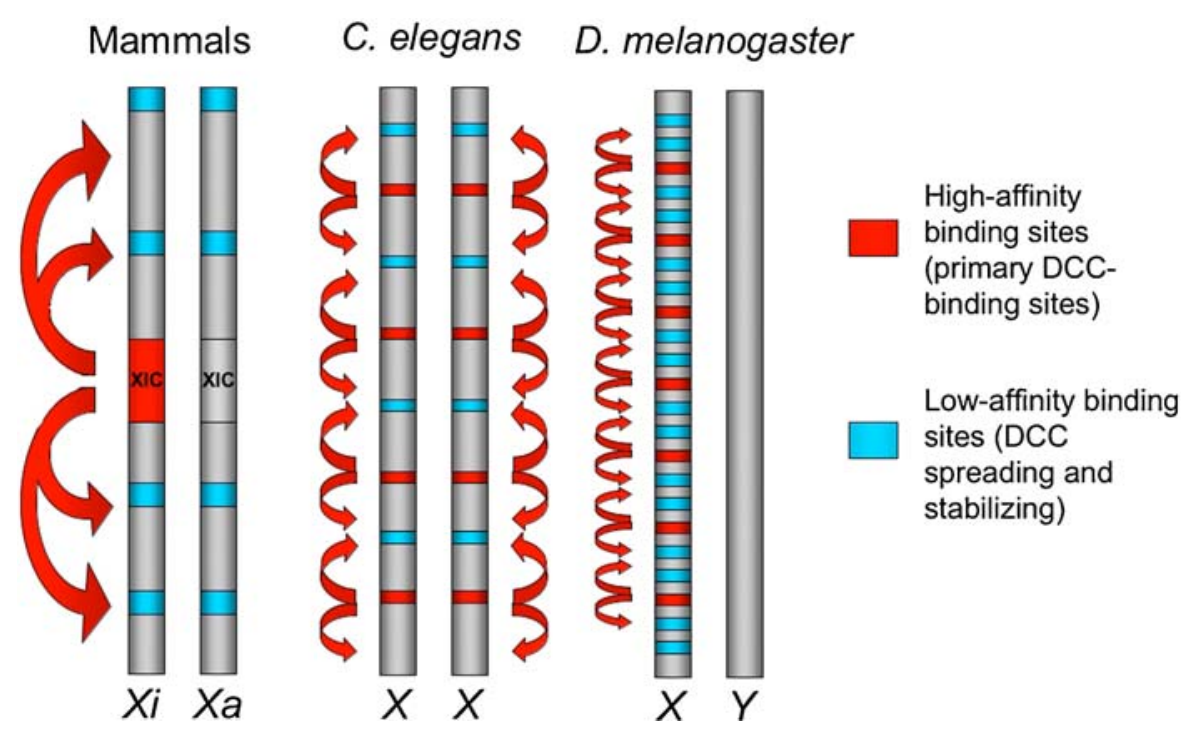

Fig. 2 Recognition of the $\mathrm{X}$ chromosome: cis-elements and spreading. In humans, a single primary targeting site-the $\mathrm{X}$-inactivation center (XIC) - serves as the entry site from which spreading of silent heterochromatin along one entire $\mathrm{X}$ chromosome occurs. In C. elegans, several primary recruitment sites occur on the X chromosome (to date, about 40 such highaffinity sites have been identified), from which spreading of the DCC in cis occurs. Two types of DCC-binding sites have been characterized experimentally in C. elegans: rex sites (recruitment elements on $\mathrm{X}$ ) and dox sites (dependent on X). rex sites - which consist of clusters of at least two small consensus motifs - are the primary binding sites of the DCC on the $\mathrm{X}$ and recruit the

translocated locus, but often also to the adjacent autosomal regions (Kageyama et al. 2001). However, many $\mathrm{X}$ chromosome segments were shown to associate normally with the DCC when translocated onto autosomes, independently of whether they contained a described DCC entry site (Fagegaltier and Baker 2004). Recent experimental work using high-resolution ChIP-seq mapping has identified many additional chromosome entry sites, and supports a two-step model of DCC recruitment to the X chromosome of Drosophila. The DCC complex is thought to first target $>150$ (and up to 300) chromatin entry sites containing specific DCC recognition elements on the $\mathrm{X}$ chromosome in males (Alekseyenko et al. 2008; Sural et al. 2008). These entry sites appear to consist of combinations of degenerate DNA motifs enriched for repetitive sequences. After this initial, sequence-specific recognition step, local spreading from entry sites in cis along the $\mathrm{X}$ chromosome is thought to lead to DCC binding to the majority of
DCC independent of their genomic location. The more frequent dox sites only bind the DCC when they are located on the X, and are thought to be responsible for the spreading of the DCC. $D$. melanogaster harbours $>150$ high affinity sites that contain a degenerate sequence motif and which are targeted by the DCC in a sequence-specific manner. After this initial recognition step, local spreading from entry sites in cis along the X leads to DCC binding to the majority of actively transcribed genes on the $\mathrm{X}$ chromosome. Although secondary binding signals on the X may play a role in Drosophila, cis spreading occurs primarily through the recognition of H3K36-methylated nucleosomes (a general features of transcribed genes)

actively transcribed genes on the $\mathrm{X}$ chromosome (Alekseyenko et al. 2008). Although secondary binding signals on the $\mathrm{X}$ may also play a role, cis spreading to the majority of X-linked genes occurs primarily through the recognition of H3K36methylated nucleosomes (a general features of transcribed genes) by MSL-3, and is therefore largely independent of further sequence signals (Larschan et al. 2007; Sural et al. 2008).

Targeting of the $\mathrm{X}$ by the C. elegans DCC follows a similar approach to Drosophila, where DCCrecruiting signals of different affinities occur along the $\mathrm{X}$ chromosome. However, there appears to be a lower density of these binding sites on the $\mathrm{X}$ of $C$. elegans compared to Drosophila, implying spreading of the DCC over larger physical distances along the X chromosome in C. elegans (Fig. 2). Some large sections of the $C$. elegans $\mathrm{X}$ chromosome do not recruit the DCC when translocated to autosomes despite being associated with the DCC and normally 
dosage-compensated when X-linked (Meyer 2005). This suggests that their association with the DCC in their native $\mathrm{X}$ location results from spreading from flanking DCC-binding sequences over significant physical distances. Thus, a plausible model of $\mathrm{X}$ recognition in $C$. elegans involves widely spaced cisacting DCC entry sites and significant spreading from these sites to coat the entire chromosome. Consistent with this model, two types of DCC-binding sites have been characterized experimentally in C. elegans (Jans et al. 2009): rex sites (recruitment elements on X) and dox sites (dependent on $\mathrm{X}$ ). rex sites are the primary binding sites of the DCC on the $\mathrm{X}$ and consist of clusters of at least two small consensus motifs (McDonel et al. 2006). They recruit the DCC independent of their genomic location. The more frequent $d o x$ sites only bind the DCC when they are located on the $\mathrm{X}$, and are thought to be responsible for the spreading of the DCC. They do not share the consensus motifs of rex sites, but do show an enrichment for a different G-rich 18bp motif (Jans et al. 2009). Since dox sites do not have the sequence signals required to independently recruit the DCC, it is possible that, like in Drosophila, other properties of the chromatin play a role in the secondary binding of the DCC (Jans et al. 2009).

What can we learn from these three examples of mechanisms of dosage compensation? First, as predicted since the sixties but only recently documented experimentally, all dosage compensation mechanisms operate to increase the expression of X-linked genes in males (instead of equalizing expression of X-linked genes between the sexes). If this is achieved by up-regulating X-linked expression through the action of a male-specific DCC, as in Drosophila, no further modulation of expression is necessary. If the expression of X-linked genes is up-regulated in a nongender-specific manner, a second pathway is required to secondarily down-regulate expression in females.

A second interesting point is that, although they are not homologous, use different genes, and ultimately work in opposite ways, the $C$. elegans and $D$. melanogaster dosage compensation pathways share several characteristics. In these organisms, dosage compensation is achieved by means of sex-specific DCCs that originated through co-opting of genes involved in sex-determination. This is particularly visible in C. elegans, where the two pathways overlap repeatedly. In Drosophila, the $S x l$ gene, an RNA splicing enzyme, is expressed specifically in $\mathrm{XX}$ embryos, where it initiates the cascade leading to the female phenotype. One of the targets of $S x l$ is the mRNA of $m s l-2$, a protein required for DCC assembly. In the presence of $S x l$, the $m s l-2$ mRNA is spliced into an inactive form, which effectively inhibits the formation of the DCC in females (Bashaw and Baker 1995; Kelley et al. 1995; Zhou et al. 1995). The specific targeting of the $\mathrm{X}$ chromosome in these two species is also similar, with initial binding of the DCC to high affinity entry points on the $\mathrm{X}$, followed by spreading to adjacent regions, possibly assisted by secondary, lower affinity binding sites. This contrasts sharply with mammalian dosage compensation, which has neither a sex-specific DCC nor chromosomespecific targeting; instead, all chromosomes carrying the XIC locus are targeted, and it is the number of $\mathrm{X}$-chromosomes in the cell that initiates Xist expression, not the sex in which they are located. These differences between mammals and C.elegans/D.melanogaster may reflect differences in the evolutionary history of their DCC; we discuss this possibility below.

The role of non-coding RNAs should also be emphasized, as they play a key role in the targeting of the $\mathrm{X}$ in both mammals and Drosophila. This is in line with recent literature highlighting the importance of non-coding RNAs for the regulation of gene expression (Mattick et al. 2009), although most of the known examples concern gene silencing, whereas in Drosophila the roX genes are used for upregulation. Finally, once the mechanisms that control $X$ chromosome upregulation in mammals and nematodes are characterized, it is well possible that further characteristics common to all three organisms will be identified.

\section{The evolutionary origin of dosage compensation genes: recruitment of pre-existing regulatory complexes}

As discussed above, dosage compensation differs greatly between the various organisms under study, and involves different genes. With the availability of whole genome sequences of several fly, nematode and mammalian species, another important question can be addressed: how are protein complexes created and recruited to perform newly evolved functions, such as dosage compensation in response to $\mathrm{Y}$ degeneration? While the mechanics of dosage compensation differs 
markedly between animal lineages, in each wellstudied case it involves the recruitment of a preexisting chromatin regulatory system to modulate expression of an entire $\mathrm{X}$ chromosome. The proteins that participate in these chromatin-modifying complexes appear to have an ancestral association that predates their role in dosage compensation. Thus, the evolution of dosage compensation does not require the emergence of novel regulatory proteins but instead the evolution of a recruiting mechanism that targets pre-existing regulatory complexes to a specific chromosome.

Several proteins of the Drosophila DCC have conserved homologs in nematodes, mammals and even in yeast (Hilfiker et al. 1997; Eisen et al. 2000). A complex that contains all $\mathrm{msl}$ protein homologs but one (mle, which may have gone undetected since it appears to have a more peripheral association with the complex in flies) has been detected in mammals (Smith et al. 2005). The human MSL-complex homolog is also involved in acetylating histone $\mathrm{H} 4$ at lysine 16, but binds to all chromosomes, and in both sexes, suggesting that a housekeeping complex present ancestrally was co-opted for dosage compensation in Drosophila. MOF, the DCC protein directly responsible for the acetylation of histone $\mathrm{H} 4$, has recently been found to perform the same function on the promoter region of genes located on the $\mathrm{X}$ and autosomes of both sexes in Drosophila (Kind et al. 2008). It therefore appears possible for an epigenetic pathway to be recruited for dosage compensation while maintaining its original regulatory function. The recruitment of this epigenetic complex for male-specific up-regulation of X-linked genes in Drosophila is likely to have occurred relatively rapidly, as DCC-protein homologs are not involved in dosage compensation of another Dipteran insect, Sciara ocellaris (Ruiz et al. 2000), although the MSL complex has been shown to mediate dosage compensation in several other Drosophila species. An interesting recent discovery regarding chromosomewide regulation in fruit flies involves an autosomespecific protein, POF (Painting of Fourth). POF is a putative RNA binding protein that specifically binds the tiny fourth chromosome of both sexes in $D$. melanogaster (Larsson and Meller 2006). Surprisingly, in D. busckii-a species in which the fourth chromosome has become fused to the ancestral $\mathrm{X}$ chromosomes- $P O F$ paints the entire $\mathrm{X}$ chromosome in males only, suggesting that POF is participating in dosage compensation, while no MSL binding to the male X was detected (Larsson et al. 2000). This suggests that an alternative system of dosage compensation may be used even within the genus Drosophila. Haddrill et al. (2007) found that genes located on the D. melanogaster fourth chromosome have, on average, higher levels of expression than genes on other chromosomes, which they interpret as a response to reduced gene activity caused by the accumulation of deleterious mutations on this non-recombining chromosome. However, increased expression of genes on chromosome four could also result from their association with POF, if POF-related mechanisms were ancestrally responsible for achieving dosage compensation in Drosophila.

Unlike the $m s l$ proteins, roX1 and roX2 are not present in the human MSL-complex. The non-coding RNAs have been shown to be essential for specific DCC binding to the $\mathrm{X}$ chromosome (Li et al. 2008), indicating that the recruitment of the $\operatorname{ro} X$ genes to the MSL-complex may have been a crucial step in the acquisition of dosage compensation in Drosophila. The origin of roX1 and roX2, however, remains a mystery, as no homologs have been found in the genome of the Dipteran insect Anopheles gambiae (Inagaki et al. 2005). Even within the genus Drosophila, not all species appear to have both $r o X$ genes (Park et al. 2007). Since $r o X 1$ and $r o X 2$ are functionally redundant in D. melanogaster, this may not be surprising, and identifying non-coding genes based on sequence conservation poses a general difficulty. However, despite low homology between roX genes identified in various Drosophila species, male-specific expression and $\mathrm{X}$ chromosome-specific binding of roX genes are conserved (Park et al. 2007).

A similar recruitment of ancestral epigenetic mechanisms that regulate chromatin compaction to perform dosage compensation functions occurred in C. elegans. In this species, three proteins of the DCC (DPY-26, DPY-27 and DPY-28) show similarity to proteins of the 13S condensin (Chuang et al. 1994; Lieb et al. 1996; Hagstrom and Meyer 2003), a mitotic complex involved in chromatin compaction and nucleosome resolution that is conserved from prokaryotes to eukaryotes (Hirano 1999). Another protein of the DCC, MIX-1, is a member of both the DCC and the $13 \mathrm{~S}$ condensin complex (Lieb et al. 1998; Hagstrom et al. 2002). In addition to being similar to a condensin protein, DPY-28 participates in crossing 
over regulation in the germline, further highlighting the dual function of some DCC proteins (Tsai et al. 2008). Additional DCC subunits confer sex specificity to the dosage compensation process and recruit the condensin-like DCC subunits to X chromosomes.

Understanding the origin of Xist and Tsix proved challenging, as these non-coding RNAs are found only in eutherian mammals (Duret et al. 2006), where they are conserved (Chureau et al. 2002). Recently, a region homologous to the mammalian XIC region has been identified in the chicken genome (Duret et al. 2006). Although the genes flanking the XIC were found to be syntenic in these species, no RNA gene was found to correspond to Xist; instead, a protein coding gene of unknown function, $\operatorname{Lnx3}$, was identified in the expected Xist region. Further analysis showed that, although Xist is much larger than $\operatorname{Ln} x 3$ and contains no protein coding sequence, some of its exons present significant similarity to $\operatorname{Ln} x 3$, indicating that Xist originated from Lnx3 (Duret et al. 2006). This has occurred at least partly through the acquisition of additional transposable-element-derived exons (Elisaphenko et al. 2008). Similarly, other XIC genes appear to have evolved by pseudogenization of coding genes of the ancestral XIC region (Elisaphenko et al. 2008).

Several lines of evidence suggest that transposable elements (TEs) played an important role in the acquisition of dosage compensation. As mentioned above, several XIC genes evolved by accumulating TE repeats in their coding sequence. Lyon (1998) also postulated that LINE elements may play a role in the spreading and stabilizing of the Xist RNA on the X chromosome, a theory supported by the higher frequency of LINE elements on the human $\mathrm{X}$ than on the autosomes, particularly around the XIC (although this accumulation of LINE elements is not detected in the mouse XIC region). A direct demonstration that dosage compensation and transposable elements are closely related in Drosophila was provided by Matyunina et al. (2008), who showed that MOF, one of the DCC proteins of Drosophila, is also involved in the repression of the copia LTR retrotransposon. This close relation between transposable elements and dosage compensation may reflect the history of epigenetic mechanisms, which are thought to have originated primarily as a defence against transposable elements (Matyunina et al. 2008).
The evolution of dosage compensation: dynamic interactions between $Y$ degeneration and dosage compensation

Although the mechanisms underlying dosage compensation are well studied, understanding how they arise, and the steps by which they arise, will require further investigations. A longstanding question in the study of how dosage compensation evolved has been whether the regulation of X-linked gene expression occurs independently for each gene, or whether large blocks of the $\mathrm{X}$ chromosome become dosagecompensated simultaneously (Fig. 3). Resolving this question has several important implications. The first concerns the different models of Y-chromosome degeneration, as they may predict different steps in the evolution of dosage compensation, depending on their underlying evolutionary parameters (Charlesworth 1996). If the $Y$ chromosome degenerates primarily through "hitchhiking" of strongly deleterious mutations when beneficial variants are swept to fixation, each of these sweeps will only carry a relatively small number of deleterious mutations at few genes to fixation. If such selective sweeps fixing strongly deleterious mutations only occur sporadically, then this model of Y-degeneration may predict that dosage compensation should evolve on a gene-by-gene basis (Fig. 3). If the $\mathrm{Y}$ degenerates mostly as a result of inefficient removal of mildly deleterious mutations from the population - or if recurrent selective sweeps are common and fix a large number of mildly deleterious alleles simultaneously-all genes on a proto-Y will decay at similar rates, but individual genes will only be impaired very slightly. Under this scenario, selection may not be strong enough to acquire dosage compensation at individual genes, but instead the entire $\mathrm{X}$ will be under selective pressure to increase gene expression, leading to large blocks of the $\mathrm{X}$ being compensated at once (Fig. 3). The relative importance of a few, strongly deleterious mutations versus many weakly deleterious mutations in causing $\mathrm{Y}$ degeneration is not known, and depends on many poorly known population parameters, such as the mutation rate for both beneficial and deleterious alleles, and their underlying distributions of selective effects.

In addition, the temporal dynamics of $\mathrm{Y}$ degeneration is expected to change over time, due to-among other factors - changes in the number of functional 

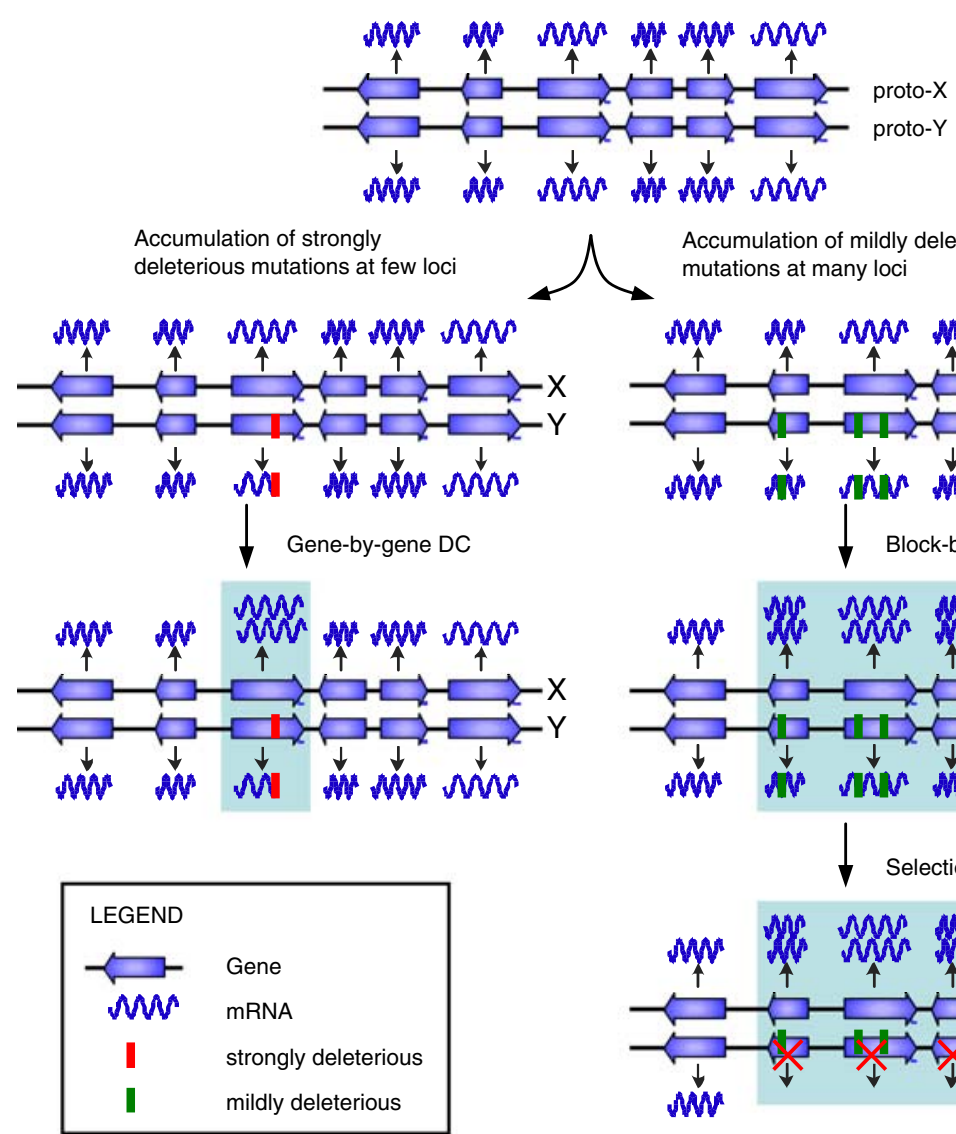

Accumulation of mildly deleterious mutations at many loci
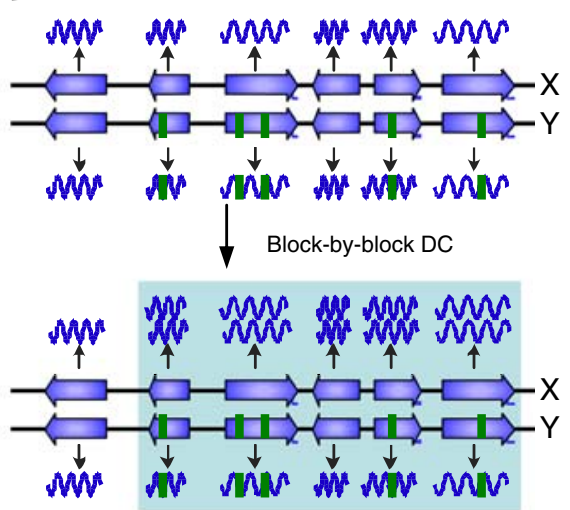

Selection for $\mathrm{Y}$ inactivation?

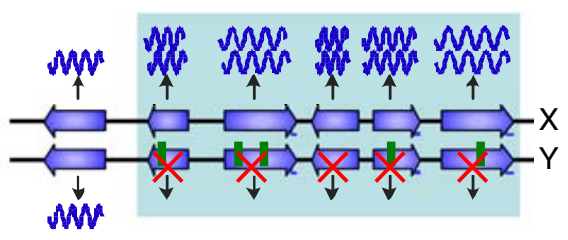

Fig. 3 Gene-by-gene or block-by-block acquisition of dosage compensation. As proto-Y-chromosomes accumulate deleterious mutations, genes become randomly mal-adapted or inactivated on the proto-Y. Dosage compensation can either evolve on a gene-by-gene basis, i.e. whenever a gene becomes inactive its homolog on the $\mathrm{X}$ becomes dosage compensated. Alternatively, once a certain number of genes become mal-adapted or inactive on the proto- $\mathrm{Y}$, blocks of genes on the $\mathrm{X}$ become dosage compensated simultaneously. Different models of $Y$ degeneration may drive the evolution of different modes of

genes on a degenerating Y chromosome. It has been shown that this reduction in functional genes can result in different processes of $\mathrm{Y}$ degeneration dominating different evolutionary stages in the transition of a gene-rich proto-Y chromosome into a degenerate Y (Bachtrog 2008a). In particular, purifying selection to remove mildly deleterious mutations from the population may be particularly ineffective on a young, gene-rich $\mathrm{Y}$ chromosome, while selective sweeps driving the fixation of more strongly deleterious mutations may become more important at later stages of $\mathrm{Y}$ degeneration (Bachtrog 2008a). This could favour a block-model of dosage-compensation

dosage compensation. In particular, Y-degeneration driven by the accumulation of strongly deleterious mutations at few loci may favour dosage-compensation mechanisms to evolve on a gene-by-gene basis, while the accumulation of many weakly deleterious mutations may select for dosage compensation at the level of blocks of genes. If blocks of genes become dosage compensated simultaneously on the $\mathrm{X}$ chromosome, this could in turn result in selective pressure to down-regulate maladapted or even fully functional genes on the $\mathrm{Y}$, in order to restore proper expression balance for these genes in females

in the early stages of sex chromosome evolution, and select for genes becoming compensated individually later on. Again, however, the exact temporal dynamics of Y degeneration depends on many poorly known evolutionary parameters, making quantitative predictions on how we would expect dosage compensation to evolve difficult.

Although both types of interference effects are likely to participate in the degeneration of the $\mathrm{Y}$ chromosome after recombination is repressed, Charlesworth (1996) pointed out that different populations might be affected to different extents by the two effects depending on their effective population 
size. Species with smaller effective population sizes, such as mammals, are likely to be strongly affected by the accumulation of moderately deleterious mutations that are not being efficiently removed from the population. Flies and nematodes, on the other hand, have much larger effective population sizes (the current effective population size of $C$. elegans is quite small, but evidence suggests that this is due to a recent reduction in effective population size from a large ancestral population; Cutter 2006) and may therefore have evolved to a greater extent through the hitchhiking of more strongly deleterious mutations together with beneficial alleles. As pointed out above, mammalian dosage compensation does indeed have characteristics that set it apart; interestingly, X-inactivation seems to be primarily a chromosome-wide mechanism, with a few genes escaping X-inactivation. Dosage compensation in $C$. elegans and D. melanogaster appears to be much more localized, with a multitude of binding sites located along the $\mathrm{X}$. This is in agreement with this theory of many weakly deleterious mutations accumulating on the proto- $Y$ chromosome in mammals at many genes, selecting for large blocks on the $\mathrm{X}$ to be compensated simultaneously, while more strongly deleterious mutations fixing by hitchhiking with beneficial alleles in C. elegans and Drosophila select for more localized mechanisms of dosage compensation.

It should be noted, however, that it is of course possible that the repression of X-linked expression in mammalian females initially evolved on a gene-by-gene basis, and that a chromosome-wide mechanism of $\mathrm{X}$-inactivation was secondarily adopted. Furthermore, nothing is known about the molecular basis for the initial up-regulation of $\mathrm{X}$ expression in mammals affecting both sexes, which may also be regulated independently for each gene. However, it appears sensible for the two mechanisms (up-regulation of the $\mathrm{X}$ in both sexes, and inactivation of the $\mathrm{X}$ in females) to evolve in parallel, and chromosome-wide mechanisms for up-regulation of $\mathrm{X}$ genes in mammals are supported by experimental data (Nguyen and Disteche 2006).

In general, mechanisms of dosage compensation that have been well-established for millions of years only provide limited information on how they were created in the first place. An approach that has proved useful in understanding the early steps of sex chromosome evolution consists in studying sex chromosomes that have only recently evolved. These can fall into two categories: true newly evolved sexchromosomes, and autosomes that have become fused to sex-chromosomes (neo-sex chromosomes). Several neo-X chromosomes in the genus Drosophila have evolved dosage compensation over some or all of their length, by co-opting the existing dosage compensation machinery. For example, D. miranda has a neo-sex chromosome system that was formed only about 1 million years ago, and roughly half of the genes originally present on the neo-Y are already degenerate (Bachtrog et al. 2008). This massive degeneration of gene function on the neo-Y (involving over 1000 genes) has triggered an evolutionary response in its former homolog, the neo-X, which is already partly recruiting the molecular machinery necessary for dosage compensation. Targeting of the DCC to the neo-X chromosome of $D$. miranda must have involved the recent (adaptive) fixation of many de novo binding sites for the dosage compensation machinery. Interestingly, rates of adaptive evolution on the neo-X chromosome of $D$. miranda were shown to be about 10-fold higher than background levels of adaptation in the genome (Bachtrog et al. 2009), consistent with the action of positive selection at many genomic regions to acquire binding sites for the DCC on the neo-X.

The mode of how dosage compensation evolved in response to mutation accumulation on the $\mathrm{Y}$ can potentially affect the process of $\mathrm{Y}$ degeneration itself (Fig. 3). In particular, if dosage compensation evolves in a block-by-block manner, large segments of a proto$\mathrm{Y}$ chromosome may become functionally redundant. Both genes which have only very slightly impaired functions and genes that are fully functional on the $\mathrm{Y}$ chromosome could become dosage compensated on the $\mathrm{X}$ and may therefore decay neutrally on the $\mathrm{Y}$. Thus, under a block-model of dosage compensation, the rate of $\mathrm{Y}$ degeneration will be greatly increased once a genomic region acquires dosage compensation. In fact, under this scenario it may actually become beneficial to down-regulate either mal-adapted or fully functional genes on a proto- $Y$ chromosome which are dosage compensated on the $\mathrm{X}$, to restore proper gene dose for these genes in males. Genes could become down-regulated individually by mutations in their regulatory sequences or by transposable element insertions, or large regions of evolving $\mathrm{Y}$ chromosomes could become transcriptional silenced simultaneously by epigenetic modifications, such as 
heterochromatinization. Thus, under a block-by-block model of dosage compensation, $\mathrm{Y}$ degeneration, at some time point, will become an adaptive process. Interestingly, the neo-Y chromosome of $D$. miranda has been shown to have undergone recent positive selection (Bachtrog 2004). While it is of course possible that some genes evolved male-beneficial functions, this could also reflect adaptive downregulation of neo-Y genes that are dosage compensated on the neo-X. Further studies of sex chromosomes that are in the process of acquiring dosage compensation will help uncover the dynamic interactions between $\mathrm{Y}$ degeneration and the evolution of dosage compensation.

\section{The glitch in the theory: lack of dosage compensation in $\mathrm{ZW}$ systems}

We have focused so far on the most frequent sexchromosome system, male heterogamety. Female heterogamety (males are ZZ, females are ZW), the system found in birds, moths and butterflies, provides an interesting complement to the previous studies, as it allows us to examine separately the consequences of always being transmitted through males (which often have, for instance, a higher mutation rate) from other predictions of sex chromosome evolution theory that are expected to affect both systems equally. In agreement with theories of sex chromosome evolution (see above), $\mathrm{W}$ chromosomes are very similar to $\mathrm{Y}$ chromosomes: they contain few functional genes and are mostly heterochromatic, have low sequence diversity and high rates of non-synonymous divergence compared with the autosomes (Berlin and Ellegren 2005; Berlin et al. 2007; Vitkova et al. 2007). Since most genes on Z-chromosomes lack a homolog on the $\mathrm{W}$, these same theories therefore predict that Z-linked genes should also be dosagecompensated in females.

An early examination of chromatin condensation showed no difference between $\mathrm{Z}$ chromosomes and autosomes in birds (Cock 1964), which suggested absence of dosage compensation (in male heterogametic systems, dosage compensation is detectable by differences in chromatin compaction of dosagecompensated X chromosomes). Small-scale studies of gene expression at few loci in birds and butterflies reached similar conclusions (Johnson and Turner
1979; Baverstock et al. 1982), leading to the puzzling suggestion that ZW systems may not have evolved dosage compensation.

More recently, female and male expression levels were systematically compared in birds using microarray studies (chicken and zebrafinch) and a few Z-linked genes were in fact shown to be dosage compensated. However, the main conclusion of these genome-wide studies remained that in birds dosage compensation seems to be the exception rather than the rule, and most Z-linked genes are expressed at significantly higher levels in males than females (Ellegren and Parsch 2007; Arnold et al. 2008). A recent expression analysis of over 500 genes located on the Bombyx mori (silkworm) Z chromosome detected a similar pattern in this species, with a vast majority of Z-linked genes presenting male-biased expression levels (Zha et al. 2008).

Several explanations have been put forward to account for this puzzling observation. Since not all genes function in a dosage dependent manner, some organisms with heteromorphic sex chromosomes will simply not require the development of chromosomewide mechanisms of dosage compensation, independently of which sex is heterogametic (Graves and Disteche 2007). In particular, the necessity of evolving dosage compensation may depend on both the types of genes initially present on the sex chromosomes, and the number of genes located on the degenerating Y. Experimental data suggest that hemizygosity at a few genes or small genomic regions is usually tolerated, while hemizygosity over extended segments or entire chromosomes is lethal. If $\mathrm{Z}$ chromosomes of birds and Lepidoptera are generally gene-poor, a lack of dosage compensation in these species could simply result from sufficient buffering of the genome against small regions of hemizygosity, accompanied by individual up-regulation of the few genes that are dosage-sensitive. $\mathrm{X}$ chromosomes of Drosophila, C. elegans and humans are gene-rich, harbouring roughly 2300, 3100, and 1500 genes, respectively. The $\mathrm{Z}$ chromosomes of both Bombyx and chicken contain slightly fewer genes, with roughly 840 and 600 protein-coding genes identified on the chicken and the silkworm $\mathrm{Z}$ chromosome, respectively. However, hemizygosity of several hundred genes is usually lethal in Drosophila, suggesting that the lack of dosage compensation in chicken and Bombyx does not simply result from a low gene 
number on the Z. Another possibility is that these ZW pairs are still in the early stages of dosage compensation evolution, after up-regulation of the $\mathrm{Z}$ in both sexes but before the increase in expression of $X$ - or Z-linked genes becomes sex-specific (see above). Data obtained for birds, however, do not support this idea: first, the $\mathrm{Z}$ and $\mathrm{W}$ chromosomes of birds diverged approximately at the same time as the mammalian $\mathrm{X}$ and $\mathrm{Y}$ (over a hundred million years ago), and before the Drosophila sex chromosomes. Second, the Z/Autosomal ratio of expression in chicken and zebrafinch males is approximately 1 (Ellegren et al. 2007; Itoh et al. 2007), which is not consistent with an chromosome-wide increase of expression on the $\mathrm{Z}$.

Other theories, including sexual antagonism, malebiased mutation rates and increased egg size in $\mathrm{ZW}$ species, view the lack of dosage compensation as a consequence of female heterogamety. (a) Sexual antagonism: It has been shown theoretically and empirically that sexual selection, as well as sexspecific selective forces, can lead to different accumulation of genes with sex-specific functions on the sex chromosomes (Rice 1984). Thus, it is possible that Z-linked genes are expressed at higher rates in males than in females not because dosage compensation is inefficient, but because these are genes with male functions. Sexual selection theory suggests that male secondary characters would more likely be established in species with male homogamety (i.e. ZZ males) than those with male heterogamety (i.e. XY males; Reeve and Pfennig 2003; Albert and Otto 2005), and comparative analysis among a list of taxa suggests that male secondary characters are more exaggerated in ZZ/ZW systems than in $\mathrm{XX} / \mathrm{XY}$ systems (Reeve and Pfennig 2003). In addition, avian Z-linked genes show evidence of accelerated rates of functional evolution, while no such effect was found for X-linked genes in mammals (Ellegren 2009). Thus, the higher levels of sex-linked gene expression in males may favour Z-linkage of genes under sexual selection, and this effect may be more pronounced in $\mathrm{ZZ/ZW}$ genetic systems than $\mathrm{XX} / \mathrm{XY}$ systems. (b) Male-biased mutation: In many organisms, the production of female and male gametes requires different numbers of cell divisions. Since most mutations occur during cell division, this can lead to differences in male and female mutation rates. A male-biased mutation rate has been observed in mammals and birds, and is associated with increased mutation rates on the $\mathrm{Y}$, while female-limited $\mathrm{W}$ chromosomes have lower rates of mutation than other chromosomes (Miyata et al. 1987). This effect could slow down the degeneration of the $\mathrm{W}$ chromosome, allowing for sexspecific dosage regulation to evolve on a gene by gene basis. Although the role of mutation rates on the degeneration of $\mathrm{Y}$ chromosomes has been quantified (Engelstädter 2008), the extent to which this affects ZW systems remains to be determined. This argument is further complicated by the fact that in many species (including birds and mammals), recombination between the proto-sex chromosomes is not restricted simultaneously over their entire length, but instead different genomic regions along the evolving sex chromosomescontaining only a subset of the genes - stop recombining at different evolutionary time points. Reducing the number of genes within the non-recombining segments of the proto-sex chromosomes has the same effect as reducing the chromosome-wide mutation rate on an evolving Y (or W) chromosome, thereby also reducing the speed of Y degeneration (Bachtrog 2008a). Thus, the effect of reduced mutation rates in ZW systems is confounded by the number of genes present in each non-recombining strata of an evolving sex chromosome pair, of which we only have limited knowledge. (c) Megalecithal eggs: Female-heterogametic species tend to have large eggs (e.g. birds) that contain large amounts of maternal mRNAs. Chandra (1991) pointed out that this could counteract the loss of W-linked genes that are required for early development, because females can produce and store large quantities of these mRNAs in the egg and transmit them to embryos. In species with smaller eggs, such as Drosophila, maternal RNAs are also crucial for zygotic and immediate postzygotic development but are soon replaced by locally produced mRNAs; the abundance of maternal mRNAs of ZW organisms may be sufficient for embryonic transcription to be put on hold until morphogenesis has been determined, thereby avoiding dosage problems at this crucial stage (Chandra 1991). This theory assumes that gene dose is more crucial at early stages of development, an assumption that has not been explicitly tested.

The extent to which each of these hypotheses affects the evolution of $\mathrm{Z}$ chromosomes remains to be determined. Furthermore, since male and female expression levels of Z-linked genes have only been analysed globally in two independently evolved ZW 
systems, it is unclear if the lack of dosage compensation in birds and butterflies is a coincidence or if it reflects true differences in the evolution and physiology of ZW organisms. Further studies of female heterogametic taxa - as found in many reptile specieswill help clarify this issue. Additionally, little is known about dosage compensation in plants that have heteromorphic sex chromosomes. Plants may generally be more tolerant to gene dose imbalances (for example, they are often polyploid and many genes occur in gene families), suggesting that they could also lack dosage compensation mechanisms. Future studies on levels of gene expression in plants with heteromorphic sex chromosomes will be of great interest to determine the extent of dosage compensation in these systems.

\section{Dosage compensation and the evolution of well-established sex chromosomes}

Most of the work reviewed here concerns the early evolution of sex chromosomes and the acquisition of dosage compensation mechanisms to counter-balance gene dose deficiencies caused by the degeneration of the Y chromosome. It is, however, worth noting that dosage compensation can also affect the current evolution of established sex chromosomes, both at sequence divergence and gene movement levels, and this may provide an explanation for some of the peculiar patterns of evolution observed on $\mathrm{X}$ chromosomes (Vicoso and Charlesworth 2006).

The presence of multiple DCC-binding sites along the X chromosomes of Drosophila and Caenorhabditis is likely to constrain the evolution of non-coding sequence. If binding sites are conserved within these clades, the accumulation of mutations that disrupt them will be prevented, so that non-coding sites are expected to evolve more slowly on the $\mathrm{X}$ chromosome than on autosomes. The extent of this bias clearly depends on the total number and size of dosage compensation binding sites along the $\mathrm{X}$, and how easily DCC-binding sites can be lost and gained in the genome (cis binding sites often show surprisingly fast rates of turn-over between species). An unexpected finding that emerged from $D$. melanogaster polymorphism studies is that there is widespread species-specific positive selection on $\mathrm{msl}$ genes in this taxa (Rodriguez et al. 2007). The DNA- binding region of the MSL complex, in particular, has accumulated several adaptive changes, and it has been suggested that this may be coupled with adaptive changes at the DCC-binding sites of the X chromosome (Rodriguez et al. 2007). Indeed, a population genetics study of three well-characterized binding sites on the $\mathrm{X}$ chromosome has detected positive selection at these regions in D. melanogaster, consistent with the idea of adaptive co-evolution between the DCC proteins and their binding sites (Bachtrog 2008c). The selective pressure underlying this coevolution is unknown, but could involve some conflict scenarios such as male-killing bacteria that specifically detect components of the MSL complex or defence against transposable elements. It will be of great interest to investigate patterns of polymorphism at many more recently identified DCC binding sites on the X of D. melanogaster, to confirm the finding of adaptive co-evolution between DCC proteins and their binding sites. Furthermore, gene expression studies in hybrids may reveal whether X-linked genes are more likely to be miss-expressed relative to autosomal genes, due to incompatibilities between DCC proteins and their binding sites. Problems in dosage compensation in species hybrids could help to explain the empirical observation that in species hybrids the heterogametic sex is more likely to suffer from sterility or inviability (known as Haldane's rule).

A further confounding effect derives from the fact that X-chromosomes in several species of Drosophila tend to be more diverged at all sites, synonymous, non-synonymous and all classes of non-coding (Begun et al. 2007). It is possible that all X-linked sites are under stronger positive selection and/or weaker negative selection than the autosomes. It seems however more likely that the mutation rate on the $\mathrm{X}$ chromosome is higher than on the autosomes. As discussed before, mutation rate differences on sex chromosomes versus autosomes could reflect differences in mutation rates of males versus females (i.e. a lower mutation rate in the male germline than in the female germline could result in a higher mutation rate of the X). However, estimates of the number of cell divisions are similar for the male and female germlines of D. melanogaster (Drost and Lee 1998; but note that this estimates strongly dependent on the mean mating age of males and females which are hard to estimate in the wild). In fact, mutation rates were estimated to be higher in males of a different 
Drosophila species, D. miranda (Bachtrog 2008b). Another explanation that has been put forward is that the hypertranscription of the $\mathrm{X}$ chromosome required for dosage compensation may itself be mutagenic (Begun et al. 2007). Although the relation between transcription and mutagenesis is not completely understood, several experimental studies have shown that increased transcription can lead to increased mutation rates in prokaryotes, yeast and, more recently, mammalian cells (Hendriks et al. 2008). Increased transcription rates associated with dosage compensation are therefore plausible to be influencing the mutation rate of the $\mathrm{X}$ chromosome. It is, however, unclear if this can account for the increased divergence detected at X-linked sites in Drosophila, since in this group expression levels are negatively correlated with divergence levels, possibly because highly expressed genes are under stronger purifying selection against the accumulation of non-synonymous and synonymous mutations (Lemos et al. 2005).

Also, X chromosomes in Drosophila show a deficiency of genes with male-biased expression, and the mechanism of dosage compensation (i.e. hyper-transcription of the $\mathrm{X}$ through epigenetic modifications) could contribute to this observed deficiency. In particular, the modified chromatin structure of the $\mathrm{X}$ may directly interfere with subsequent transcriptional modification of X-linked genes in males. Male-biased gene expression originates mainly by increasing transcription of non-biased genes in males (rather than down-regulation in females), and higher expression levels may be harder to achieve on an already hyper-transcribed $\mathrm{X}$ chromosome (Vicoso and Charlesworth 2006; Vicoso and Charlesworth 2009). The $X$ chromosome in male Drosophila is encumbered by the DCC and its chromatin structure has been modified globally. This may limit subsequent transcription factor binding or chromatin remodelling, and thus inhibit further transcriptional activation, resulting in a deficiency of male-biased expression on the $\mathrm{X}$ chromosome. In fact, direct interference between chromatin remodelling complexes and the dosage compensation machinery has been reported in Drosophila. Limitations in rates of transcription have also been put forward as an explanation for the deficiency of male-biased genes on the Drosophila X (Vicoso and Charlesworth 2009). This is supported by the observation that the proportion of male-biased genes located on the $\mathrm{X}$ differs significantly between high and low expression genes, with high expression male-biased genes being located less often on the $\mathrm{X}$ than low expression malebiased genes. This is expected if limits in rates of transcription prevent the accumulation of male-biased genes on the $\mathrm{X}$, since such limitations are less likely to affect genes that are transcribed at low levels (Vicoso and Charlesworth 2009). It will be of interest to experimentally test whether dosage compensation can indeed help to explain the deficiency of malebiased genes on the Drosophila X.

Acknowledgements We are grateful to members of the Bachtrog lab and Brian Charlesworth for comments on the manuscript. This research is funded by NIH Grant GM076007 and a Sloan Research Fellowship and a David and Lucile Packard Fellowship to D.B.

Open Access This article is distributed under the terms of the Creative Commons Attribution Noncommercial License which permits any noncommercial use, distribution, and reproduction in any medium, provided the original author(s) and source are credited.

\section{References}

Albert AY, Otto SP (2005) Sexual selection can resolve sexlinked sexual antagonism. Science 310:119-121

Alekseyenko AA, Peng S, Larschan E, Gorchakov AA, Lee OK, Kharchenko P, McGrath SD, Wang CI, Mardis ER, Park PJ, Kuroda MI (2008) A sequence motif within chromatin entry sites directs MSL establishment on the Drosophila X chromosome. Cell 134:599-609

Arnold AP, Itoh Y, Melamed E (2008) A bird's-eye view of Sex chromosome dosage compensation. Annu Rev Genomics Hum Genet 9:109-127

Bachtrog D (2004) Evidence that positive selection drives Y-chromosome degeneration in Drosophila miranda. Nat Genet 36:518-522

Bachtrog D (2008a) The temporal dynamics of processes underlying $\mathrm{Y}$ chromosome degeneration. Genetics 179:1513-1525

Bachtrog D (2008b) Evidence for male-driven evolution in Drosophila. Mol Biol Evol 25:617-619

Bachtrog D (2006) Expression profile of a degenerating neo-Y chromosome in Drosophila. Curr Biol 16:1694-1699

Bachtrog D (2008c) Positive selection at the binding sites of the male-specific lethal complex involved in dosage compensation in Drosophila. Genetics 180:1123-1129

Bachtrog D, Hom E, Wong KM, Maside X, de Jong P (2008) Genomic degradation of a young $\mathrm{Y}$ chromosome in Drosophila miranda. Genome Biol 9:R30

Bachtrog, D., J. Jensen, and Z. Zhang. (2009). Accelerated adaptive evolution on a newly formed $\mathrm{X}$ chromosome. PLoS Biol

Bashaw G, Baker B (1995) The msl-2 dosage compensation gene of Drosophila encodes a putative DNA-binding 
protein whose expression is sex specifically regulated by Sex-lethal. Development 121:3245-3258

Baverstock PR, Adams M, Polkinghorne RW, Gelder M (1982) A sex-linked enzyme in birds-Z-chromosome conservation but no dosage compensation. Nature 296:763-766

Begun DJ, Holloway AK, Stevens K, Hillier LW, Poh Y-P, Hahn MW, Nista PM, Jones CD, Kern AD, Dewey CN, Pachter L, Myers E, Langley CH (2007) Population genomics: whole-genome analysis of polymorphism and divergence in Drosophila simulans. PLoS Biol 5:e310

Berlin S, Ellegren H (2005) Fast accumulation of nonsynonymous mutations on the female-specific $\mathrm{W}$ chromosome in birds. J Mol Evol 62:66-72

Berlin S, Tomaras D, Charlesworth B (2007) Low mitochondrial variability in birds may indicate Hill-Robertson effects on the W chromosome. Heredity 99:389-396

Bull JJ (1983) Evolution of Sex Determining Mechanisms. Benjamin Cummings, Menlo Park, CA

Carrel L, Willard H (2005) X-inactivation profile reveals extensive variability in $\mathrm{X}$-linked gene expression in females. Nature 434:400-404

Chandra HS (1991) How do heterogametic females survive without gene dosage compensation? J Genet 70:137-146

Charlesworth B (1978) Model for evolution of Y chromosomes and dosage compensation. Proc Natl Acad Sci USA 75:5618-5622

Charlesworth B (1996) The evolution of chromosomal sex determination and dosage compensation. Curr Biol 6:149-162

Charlesworth D (2004) Plant evolution: modern sex chromosomes. Curr Biol 14:R271-273

Chuang PT, Albertson DG, Meyer BJ (1994) DPY-27: a chromosome condensation protein homolog that regulates C. elegans dosage compensation through association with the X chromosome. Cell 79:459-474

Chureau C, Prissette M, Bourdet A, Barbe V, Cattolico L, Jones L, Eggen A, Avner P, Duret L (2002) Comparative sequence analysis of the $\mathrm{X}$-inactivation center region in mouse, human, and bovine. Genome Res 12:894-908

Cock AG (1964) Dosage compensation and sex-chromatin in non-mammals. Genet Res 5:354-365

Cutter AD (2006) Nucleotide polymorphism and linkage disequilibrium in wild populations of the partial selfer Caenorhabditis elegans. Genetics 172:171-184

Dawes HE, Berlin DS, Lapidus DM, Nusbaum C, Davis TL, Meyer BJ (1999) Dosage compensation proteins targeted to $\mathrm{X}$ chromosomes by a determinant of hermaphrodite fate. Science 284:1800-1804

Drost JB, Lee WR (1998) The developmental basis for germline mosaicism in mouse and Drosophila melanogaster. Genetica 102(103):421-443

Duret L, Chureau C, Samain S, Weissenbach J, Avner P (2006) The Xist RNA gene evolved in Eutherians by pseudogenization of a protein-coding gene. Science 312:1653-1655

Eisen A, Utley RT, Nourani A, Allard S, Schmidt P, Lane WS, Lucchesi JC, Côté J (2000) The Yeast NuA4 and Drosophila MSL complexes contain homologous subunits important for transcription regulation. J Biol Chem 276:3484-3491

Elisaphenko EA, Kolesnikov NN, Shevchenko AI, Rogozin IB, Nesterova TB, Brockdorff N, Zakian SM (2008) A dual origin of the Xist gene from a protein-coding gene and a set of transposable elements. PloS One 3:e2521
Ellegren H (2009) Genomic evidence for a large-Z effect. Proc Biol Sci 276:361-366

Ellegren H, Parsch J (2007) The evolution of sex-biased genes and sex-biased gene expression. Nat Rev Genet 8:689-698

Ellegren H, Hultin-Rosenberg L, Brunstr^m B, Dencker L, Kultima K, Scholz B (2007) Faced with inequality: chicken do not have a general dosage compensation of sex-linked genes. BMC Biol 5:40

Engelstädter J (2008) Muller's Ratchet and the degeneration of Y chromosomes: a simulation study. Genetics 180:957-967

Engelstädter J, Haig D (2008) Sexual antagonism and the evolution of X chromosome inactivation. Evolution 62:2097-2104

Fagegaltier D, Baker BS (2004) X chromosome sites autonomously recruit the dosage compensation complex in Drosophila males. PLoS Biol 2:e341

Graves JAM, Disteche CM (2007) Does gene dosage really matter? J Biol 6:1

Gupta, V., M. Parisi, D. Sturgill, R. Nuttall, M. Doctolero, O. K. Dudko, J. D. Malley, P. S. Eastman, and B. Oliver. (2006). Global analysis of X-chromosome dosage compensation. J. Biol. 5:3.1-3.10

Guttman D, Charlesworth D (1998) An X-linked gene with a degenerate Y-linked homologue in a dioecious plant. Nature 393:263-266

Haddrill P, Halligan D, Tomaras D, Charlesworth B (2007) Reduced efficacy of selection in regions of the Drosophila genome that lack crossing over. Genome Biol 8:R18

Hagstrom KA, Meyer BJ (2003) Condensin and cohesin: more than chromosome compactor and glue. Nat Rev Genet 4:520-534

Hagstrom KA, Holmes VF, Cozzarelli NR, Meyer BJ (2002) C. elegans condensin promotes mitotic chromosome architecture, centromere organization, and sister chromatid segregation during mitosis and meiosis. Genes Dev 16:729-742

Hendriks G, Calléja F, Vrieling H, Mullenders LHF, Jansen JG, Wind ND (2008) Gene transcription increases DNA damage-induced mutagenesis in mammalian stem cells. DNA Repair 7:1330-1339

Hilfiker A, Hilfiker-Kleiner D, Pannuti A, Lucchesi JC (1997) mof, a putative acetyl transferase gene related to the Tip60 and MOZ human genes and to the SAS genes of yeast, is required for dosage compensation in Drosophila. EMBO J 16:2054-2060

Hill WG, Robertson A (1966) The effect of linkage on limits to artificial selection. Genet Res 8:269-294

Hirano T (1999) SMC-mediated chromosome mechanics: a conserved scheme from bacteria to vertebrates? Genes Dev 13:11-19

Inagaki S, Numata K, Kondo T, Tomita M, Yasuda K, Kanai A, Kageyama Y (2005) Identification and expression analysis of putative mRNA-like non-coding RNA in Drosophila. Genes Cells 10:1163-1173

Itoh Y, Melamed E, Yang X, Kampf K, Wang S, Yehya N, Van Nas A, Replogle K, Band MR, Clayton DF, Schadt EE, Lusis AJ, Arnold AP (2007) Dosage compensation is less effective in birds than in mammals. J Biol 6:2

Jans J, Gladden JM, Ralston EJ, Pickle CS, Michel AH, Pferdehirt RR, Eisen MB, Meyer BJ (2009) A condensinlike dosage compensation complex acts at a distance to control expression throughout the genome. Genes Dev 23:602-618 
Johnson MS, Turner JRG (1979) Absence of dosage compensation for a sex-linked enzyme in butterflies (Heliconius). Heredity 43:71-77

Kageyama Y, Mengus G, Gilfillan G, Kennedy H, Stuckenholz C, Kelley R, Becker P, Kuroda M (2001) Association and spreading of the Drosophila dosage compensation complex from a discrete roX1 chromatin entry site. EMBO J 20:2236-2245

Kelley, R. L., V. H. Meller, P. R. Gordadze, G. Roman, D. R.L., and M. I. Kuroda (1999) Epigenetic spreading of the Drosophila dosage compensation complex from roX RNA genes into flanking chromatin. Cell 98

Kelley, R. L., I. Solovyeva, L. M. Lyman, R. Richman, V. Solovyev, and I. Kuroda (1995) Expression of Msl-2 causes assembly of dosage compensation regulators on the $\mathrm{X}$ chromosomes and female lethality in Drosophila Cell 81:867-877

Kind J, Vaquerizas J, Gebhardt P, Gentzel M, Luscombe N, Bertone P, Akhtar A (2008) Genome-wide analysis reveals $\mathrm{MOF}$ as a key regulator of dosage compensation and gene expression in Drosophila. Cell 133:813-828

Lahn BT, Pearson NM, Jegalian K (2001) The human Y chromosome, in the light of evolution. Nat Rev Genet 2:207-216

Larschan E, Alekseyenko AA, Gortchakov AA, Peng S, Li B, Yang P, Workman JL, Park PJ, Kuroda MI (2007) MSL Complex Is Attracted to Genes Marked by H3K36 Trimethylation Using a Sequence-Independent Mechanism. Mol Cell 28:121-133

Larsson J, Chen JD, Rasheva V, Rasmuson-Lestander $\AA$, Pirrotta V (2000) Painting of fourth, a chromosomespecific protein in Drosophila. Proc Natl Acad Sci USA 98:6273-6278

Larsson J, Meller VH (2006) Dosage compensation, the origin and the afterlife of sex chromosomes. Chromosom Res 14:417431

Lemos B, Bettencourt BR, Meiklejohn CD, Hartl DL (2005) Evolution of Proteins and Gene Expression Levels are Coupled in Drosophila and are Independently Associated with mRNA Abundance, Protein Length, and Number of Protein-Protein Interactions. Mol Biol Evol 22:1345-1354

Li F, Schiemann AH, Scott MJ (2008) Incorporation of the noncoding roX RNAs alters the chromatin-binding specificity of the Drosophila MSL1/MSL2 complex. Mol Cell Biol 28:1252-1264

Lieb JD, Albrecht MR, Chuang PT, Meyer BJ (1998) MIX-1: An essential component of the $C$. elegans mitotic machinery executes $\mathrm{X}$ chromosome dosage compensation. Cell 92:265-277

Lieb JD, Capowski EE, Meneely P, Meyer BJ (1996) DPY-26, a link between dosage compensation and meiotic chromosome segregation in the nematode. Science 274:1732-1736

Lin H, Gupta V, Vermilyea MD, Falciani F, Lee JT, O'Neill LP, Turner BM (2007) Dosage compensation in the mouse balances up-regulation and silencing of X-linked genes. PLoS Biol 5:e326

Lindsley DL, Sandler L, Baker BS, Carpenter ATC, Denell RE, Hall JC, Jacobs PA, Miklos GLG, Davis BK, Gethmann RC, Hardy RW, Hessler A, Miller SM, Nozawa H, Parry DM, Gould-Somero M (1972) Segmental aneuploidy and the genetic gross structure of the Drosophila genome. Genetics 71:157-184
Liu Z, Moore P, Ma H, Ackerman C, Ragiba M, Yu Q, Pearl H, Kim M, Charlton J, Stiles J, Zee F, Paterson A, Ming $\mathrm{R}$ (2004) A primitive $\mathrm{Y}$ chromosome in papaya marks incipient sex chromosome evolution. Nature 427:348352

Lyon M (1998) X-Chromosome inactivation: a repeat hypothesis. Cytogenet Cell Genet 80:133-137

Lyon MF (1961) Gene action in the X-chromosome of the mouse (Mus musculus L.). Nature 190:372-373

Mattick JS, Amaral PP, Dinger ME, Mercer TR, Mehler MF (2009) RNA regulation of epigenetic processes. BioEssays 31:51-59

Matyunina LV, Bowen NJ, McDonald JF (2008) LTR retrotransposons and the evolution of dosage compensation in Drosophila. BMC Mol Biol 9:55

McDonel P, Jans J, Peterson BK, Meyer BJ (2006) Clustered DNA motifs mark $\mathrm{X}$ chromosomes for repression by a dosage compensation complex. Nature 444:614-618

Meyer, B. J. (2005) X-Chromosome dosage compensation. WormBook:1-14

Meyer BJ, Casson LP (1986) Caenorhabditis elegans compensates for the difference in $\mathrm{X}$ chromosome dosage between the sexes by regulating transcript levels. Cell 47:871881

Miyata T, Hayashida H, Kuma K, Mitsuyasu K, Yasunaga T (1987) Male-driven molecular evolution: a model and nucleotide sequence analysis. Cold Spring Harbor Symp Quant Biol 52:863-867

Nguyen DK, Disteche CM (2006) Dosage compensation of the active $\mathrm{X}$ chromosome in mammals. Nat Genet 38:47-53

Park S-W, Kang YI, Sypula JG, Choi J, Oh H, Park Y (2007) An evolutionarily conserved domain of roX2 RNA Is sufficient for induction of H4-Lys16 acetylation on the Drosophila X chromosome. Genetics 177:1429-1437

Park Y, Kuroda M (2001) Epigenetic aspects of X-chromosome dosage compensation. Science 293:1083-1085

Payer B, Lee JT (2008) X chromosome dosage compensation: how mammals keep the balance. Annu Rev Genet 42:733-772

Reeve HK, Pfennig DW (2003) Genetic biases for showy males: are some genetic systems especially conducive to sexual selection? Proc Natl Acad Sci USA 100:1089-1094

Rice WR (1987) Genetic hitchhiking and the evolution of reduced genetic activity of the $\mathrm{Y}$ sex chromosome. Genetics 116:161-167

Rice WR (1984) Sex chromosomes and the evolution of sexual dimorphism. Evolution 38:735-742

Rodriguez MA, Vermaak D, Bayes JJ, Malik HS (2007) Species-specific positive selection of the male-specific lethal complex that participates in dosage compensation in Drosophila. Proc Natl Acad Sci USA 104:15412-15417

Ruiz MF, Esteban MR, Donoro C, Goday C, Sanchez L (2000) Evolution of dosage compensation in Diptera: the gene maleless implements dosage compensation in Drosophila (Brachycera suborder) but its homolog in Sciara (Nematocera suborder) appears to play no role in dosage compensation. Genetics 156:1853-1865

Smith ER, Cayrou C, Huang R, Lane WS, Côté J, Lucchesi JC (2005) A human protein complex homologous to the drosophila MSL complex is responsible for the majority of histone $\mathrm{H} 4$ acetylation at lysine 16. Mol Cell Biol 25:9175-9188 
Straub T, Becker PB (2007) Dosage compensation: the beginning and end of generalization. Nat Rev Genet 8:47-57

Sural TH, Peng S, Li B, Workman JL, Park PJ, Kuroda MI (2008) The MSL3 chromodomain directs a key targeting step for dosage compensation of the Drosophila melanogaster $\mathrm{X}$ chromosome. Nat Struct Mol Biol 15:1318-1325

Tsai CJ, Mets DG, Albrecht MR, Nix P, Chan A, Meyer BJ (2008) Meiotic crossover number and distribution are regulated by a dosage compensation protein that resembles a condensin subunit. Genes Dev 22:194-211

Vicoso B, Charlesworth B (2006) Evolution on the X chromosome: unusual patterns and processes. Nat Rev Genet 7:645653

Vicoso, B., and B. Charlesworth (2009) The Deficit of MaleBiased Genes on the D. melanogaster X Chromosome Is Expression-Dependent: A Consequence of Dosage Compensation? J Mol Evol
Vitkova M, Fukova I, Kubickova S, Marec F (2007) Molecular divergence of the $\mathrm{W}$ chromosomes in pyralid moths (Lepidoptera). Chromosom Res 15:917-930

White WM, Willard HF, Van Dyke DL, Wolff DJ (1998) The spreading of $\mathrm{X}$ inactivation into autosomal material of an $\mathrm{x}$; autosome translocation: evidence for a difference between autosomal and X-chromosomal DNA. Am J Hum Genet 63:20-28

Zha X, Xia Q, Duan J, Wang C, He N, Xiang Z (2008) Dosage analysis of $\mathrm{Z}$ chromosome genes using microarray in silkworm. Bombyx mori, Insect Biochem Mol Biol

Zhou S, Yang Y, Scott M, Pannuti A, Fehr K, Eisen A, Koonin E, Fouts D, Wrightsman R, Manning J, Lucchesi J (1995) Male-specific lethal 2, a dosage compensation gene of Drosophila, undergoes sex-specific regulation and encodes a protein with a RING finger and a metallothionein-like cysteine cluster. EMBO J 14:2884-2895 